\title{
Effect of Abnormal Amino Acid Concentrations on Blood-Brain Barrier
}

Defects in catabolic pathways for amino acids lead to the accumulation in the blood of the amino acid as well as certain metabolites and frequently are associated with neurological conditions leading to mental retardation $^{1}$. Nutritional avitaminoses such as scurvy and pellagra also show severe neurological signs ${ }^{2}$. In that the enzymes responsible for amino acid degradation, such as the transaminases and decarboxylases, require vitamins as cofactors, a paucity of these substances could lead to the accumulation of amino acids in the individual with avitaminosis.

It seems plausible that the increased systemic level of some amino acids or their metabolites might alter the permeability of the blood-brain barrier and lead to the neurologic abberations noted. The present preliminary study was intended to examine the possible alteration of the blood-brain barrier by elevated levels of amino acids in the blood and to see whether pyridoxal phosphate could protect against such alteration.

Materials and methods. In a typical experiment groups of 3 mice weighing approximately $4.0 \mathrm{~g}$ were given $1 \mathrm{ml}$ i.p. injections of one of the solutions described in the Table. 1 mouse from each group was sacrificed as given in the Table and the brains removed and weighed.

The brain from individual animals was homogenized for $1 \mathrm{~min}$ with 10 volumes per $\mathrm{g}$ of brain tissue of $0.05 \mathrm{M}$ acetate buffer, $\mathrm{pH} 5.75$ in a Potter-Elvejham homogenizer. Aliquots of $0.2 \mathrm{ml}$ from each sample were plated on stainless steel planchets and counted in a Nuclear Chicago Model 183B Gas Flow Counter. Each of the experimental series was repeated 3 times. Separate aliquots of each of the above sample mixtures were chromatographed against appropriate amino acid standards on Whatman No. 1 paper for $16 \mathrm{~h}$ using the butanol-acetic acid - water $(5: 1: 4)$ solvent system. The chromatograms were then sprayed with a $0.1 \%$ ninhydrin solution containing collidine to detect the amino acids present. The ninhydrin positive spots were cut out and separately counted in the gas flow counter.

Results. From the Table it is evident that the high level of tyrosine inhibits greatly the quantity of labelled lysine found in the brain at each of the 3 time intervals tested. The addition of pyridoxal phosphate overcomes this inhibition. At $24 \mathrm{~h}$ the vitamin by itself appears to stimulate lysine uptake as well as in the presence of large quantities of tyrosine. The data indicate clearly

Lysine incorporation into total brain homogenates

Injection mixture

\section{CPM in brain homogenates} (h after injection)

$$
24
$$

$$
48
$$

96

L-Lysine-C
L-Lysine-C14, L-Tyrosine
L-Lysine-C' 14 , L-Tyrosine,
Pyridoxal phosphate
L-Lysine-C ${ }^{14}$,

$1428+41$

$1428 \pm 41$
$721 \pm 42$

$2396 \pm 155$

$2462 \pm 131$

$1886 \pm 237$

$3187 \pm 264$

$3256+259$

$1007 \pm 133$

$3296 \pm 188$

pyridoxal phosphate that the administration of a single massive dose of tyrosine depresses the amount of labelled lysine taken up by the brain for at least $96 \mathrm{~h}$. The maximal level of lysine uptake occurs at about $48 \mathrm{~h}$ after which the rate of uptake plateaus. Pyridoxal phosphate appears somewhat stimulatory towards lysine uptake into the brain at $24 \mathrm{~h}$, but this is not significantly so at 48 or $96 \mathrm{~h}$.

On paper chromatograms, although ninhydrin positive spots corresponding to essentially all the amino acids generally found in brain could be detected in aliquots of brain homogenates, only in the spot corresponding to lysine could any radioactivity be detected.

Discussion. The first 6 months of postnatal life are thought to be of prime importance in the myelination of the human brain. Any factor, metabolic or otherwise, leading to a malfunction in the growth process at this time could conceivably lead to irreparable brain damage. A number of investigators ${ }^{3-5}$ have been able to produce rats with pathological symptoms similar to those found in phenylketonuria by i.p. injections of large quantities of tyrosine or phenylalanine.

The results of the present study could have important implications on the effects of abnormally large quantities of 1 amino acid on the developing brain. The blood-brain barrier is known to have selective uptake towards various amino acids, allowing the neutral amino acids to penetrate quite readily but being relatively impermeable to the charged amino acids such as lysine. Changing the transport system of the brain towards certain amino acids during the time of its growth would have a profound effect on its subsequent development. In our studies large amounts of tyrosine appear to greatly depress lysine uptake in the brain either by further inhibiting its ability to get in or by accelerating its efflux. However, when the level of any residue in the amino acid pool of the brain becomes too low at the time of active protein synthesis, protein deficiency would occur which in turn could be responsible for mental retardation. Thus any means of reducing the concentration of the abnormally high amino acid which would subsequently restore the amino acid uptake of the brain to normal would be effective therapy. The ability of pyridoxal phosphate in relieving the decreased lysine uptake in the brain in our study may be due in part to its ability to stimulate the metabolism of tyrosine by processes such as transamination for which it is a cofactor.

Zusammenfassung. Nachweis, dass die i.p. Injektion grosser Mengen von L-Tyrosin die Permeabilität der hypothetischen "Blut-Hirn-Schranke" beträchtlich herabsetzt. Pyridoxalphosphat verhindert nicht nur diese Hemmung, sondern steigert die Absorption von L-Lysin.

\section{A. D. Goldstone and S. B. NeEdLEMAN ${ }^{\circ}$}

Department of Biochemistry, Northwestern University Medical School and V.A. Research Hospital, Chicago (Illinois 60611, USA), 11 January 1971.

J. SterN and V. Cowie, Biochem. J. 111, 9 (1969):

\& L. Paulino, Science 160, 265 (1968).

3 S. N. ShaH and C. M. MclKeAN, Fedn Proc. 27, 488 (1968)

4 J. T. R. Clarke and J. A. Lowden, Canad. J. Biochem. 47, 291 (1969).

s S. B. NeEDLEMAN and R. W. LoNGToN, in preparation.

- This work was supported by a grant to S.B.N. from the office of Navel Research No. N 00014-70-C-0151.
Weights of the total brain homogenates ranged between $0.29 \mathrm{~g}$ and $0.36 \mathrm{~g}$ over the entire experimental period. Differences in brain weight at each time interval were insignificant. 7-day-old mice
injected i.p, and sacrificed 24,48 or $96 \mathrm{~h}$ after injection. The final concentration of L-lysine- $\mathrm{C}^{14}$ was $5 \mu \mathrm{c}$, L-tyrosine, $50 \mathrm{mg}$ and pyridoxal phosphate, $2 \mathrm{mg}$. Each figure represents an average of 3 separate experiments. 